\title{
Critical Metabolic Pathways And Genes Cooperate For Epoxy Fatty Acid-Enriched Oil Production In Developing Seeds of Vernonia Galamensis, An Industrial Oleaginous Plant
}

\author{
Yan Sun \\ Shanxi Agricultural University \\ Baoling Liu \\ Shanxi Agricultural University \\ Jinai Xue \\ Shanxi Agricultural University \\ Xiaodan Wang \\ Shanxi Agricultural University \\ Hongli Cui \\ Shanxi Agricultural University \\ Xiaoyun Jia \\ Shanxi Agricultural University \\ Run-Zhi Li ( $\nabla$ rli2001@126.com ) \\ Shanxi Agricultural University https://orcid.org/0000-0002-9933-5024
}

\section{Research Article}

Keywords: Epoxy fatty acid (EFA), Vernonia galamensis, Transcriptome, Triacylglycerol, Industrial oil, Oilseed

Posted Date: December 6th, 2021

DOI: https://doi.org/10.21203/rs.3.rs-1115579/v1

License: (c) (i) This work is licensed under a Creative Commons Attribution 4.0 International License. Read Full License

Version of Record: A version of this preprint was published at Biotechnology for Biofuels and Bioproducts on February 25th, 2022. See the published version at https://doi.org/10.1186/s13068-022-02120-2. 


\section{Abstract}

Background: Vernonia galamensis, originated from Africa, is an annual oleaginous plant of Asteraceae family. As a newly-establishing industrial oil crop, this plant produces high level (> 70\%) of vernolic acid (cis-12-epoxyoctadeca-cis-9-enoic acid), an unusual epoxy fatty acid (EFA) with multiple industrial applications. Here, transcriptome analysis and fatty acid profiling from developing $V$. galamensis seeds were integrated to uncover the critical metabolic pathways responsible for high EFA accumulation and identify the targets that could be used in assembly of EFA biosynthesis pathway in existing oilseed crops.

Results: Based on oil accumulation dynamics of $V$. galamensis seeds, we harvested seed samples from three stages $(17,38$, and 45 days after pollination, DAP) representing the initial, fast and final EFA accumulation phases, and one mixed sample from different tissues for RNA-sequencing, with three biological replicates at each sample. Using Illumina platform, we have generated a total of 265 million raw cDNA reads. After filtering process, de novo assembly of clean reads yielded 67,114 unigenes with an N50 length of 1,316 nt. Functional annotation resulted in the identification of almost all genes involved in diverse lipid-metabolic pathways, including the novel fatty acid desaturase/epoxygenase, diacylglycerol acyltransferase 1 and 2, and phospholipid:diacylglycerol acyltransferases. By comparison, we found that various genes associated with acyl editing, fatty acid $\beta$-oxidation, triacylglycerol assembly and oil-body formation had greater expression levels at middle developmental stage (38 DAP), which are consistent with the fast accumulation of EFA in $V$. galamensis developing seed, implying their fundamental roles in EFA production. Additionally, we isolated some transcription factors (such as WRI1, FUS3 and ABI4), which putatively regulated the production of $V$. galamensis seed oils. The transient assay of selected genes resulted in a synergistic increase of EFA-enriched TAG accumulation in tobacco leaves. Transcriptome data were further confirmed by quantitative real-time PCR for twelve key genes in EFA biosynthesis. Finally, a comprehensive network for high EFA accumulation in V. galamensis seed was established.

Conclusions: Our results are of significance with regard to understanding the molecular mechanisms underlying the natural epoxy oil production in this plant and provide valuable reference for developing other oilseeds with high level of valued epoxy oil.

\section{Background}

Plant oils, one of the high energy density and major carbon available from nature, have long been consumed by human not only as essential nutrients but also petroleum alternatives in the industry [1]. For producing oleochemicals such as plasticizers, adhesives, soaps, coatings, paints, lubricants and polymers, plant oils have to be chemically modified. One of the most high-valued modifications is to introduce an additional epoxy group [2]. Currently, the major sources of epoxy oils are produced by chemical epoxidation of highly unsaturated oils, such as soybean and linseed oils. However, chemical synthesis of epoxy group is a relatively expensive process and emission of volatile organic compounds 
may cause the new pollution. Compared to the artificial products, natural epoxy oils represent the inexpensive, environmental friendly and renewable feedstocks that could replace petrochemicals $[3,4]$.

For many years, natural epoxy oils were only found in some wild plants from Asteraceae and Euphorbiaceae species, including Vernonia galamensis, Vernonia anthelmintica, Stokesia laevis, Crepis palaestina and Euphorbia lagascae [5]. Notably, V. galamensis is an annual herb native to Africa, which contains the highest level of epoxy oil (about 40\%), and thus is considered to be a good model for the study of epoxy oil metabolism [6]. In fact, the industrial value of natural epoxy oil arises from its high content of vernolic acid (cis-12-epoxyoctadeca-cis-9-enoic acid), an unusual epoxy fatty acid (EFA) that comprises $>70 \%$ of $V$. galamensis seed oil [7]. Like other plants that could produce EFA-enriched oils, large-scale cultivation of $V$. galamensis is limited by its poor agronomic traits [8]. Alternatively, creating genetically modified plant has been a promising strategy for natural epoxy oil production [9]. As such, identification of genes for synthesis of EFA is one of the great challenges for genetic engineering. However, the physiological and molecular mechanisms underlying the accumulation of EFA-enriched oil in $V$. galamensis seed and other source plants remain unknown.

It is now widely recognized that EFA is catalyzed by a functional variant of $\Delta 12$-fatty acid desaturase (FAD2), which is termed as epoxygenase [10]. So far, genes encoding epoxygenase have been isolated from C. palaestina (Cpal2) and S. laevis (SIEPX) $[10,11]$. Transgenic expression of Cpal2 in Arabidopsis has successfully yielded the synthesis of unusual EFA, but its content (6.2\%) is very low [11]. Yu et al evidenced that acyl-CoA:diacylglycerol acyltransferases (DGATs) from V. galamensis have strong preference for EFA-containing substrates, thus promoting the storage of large amounts of EFA into triacylglycerol (TAG) [12]. Simultaneous co-expression of SIEPX and $V$. galamensis DGAT2 (VgDGAT2) in soybean seeds could result in EFA content of $25.8 \%$, representing the maximum level achieved in engineered plants [13]. Despite that, transgenic experiments still fail to achieve high yields of desired EFA. It seems that additional factors may influence its normal accumulation in transgenic plants. Indeed, abnormally large amounts of EFA are esterified to PC (membrane lipids) in these engineered plants [13], while EFA in PC is very low in native source plants where most of EFA is stored in TAG. Similar phenomena were also reported for transgenic production of other unusual fatty acids (FAs), such as hydroxy and conjugated FAs $[14,15]$. Excessive accumulation of unusual EFA in membrane lipid often causes detrimental phenotype for transformed tissues. These results indicate that common oilseeds may lack specialized enzymes which can transfer the unusual EFA from the site on PC to their storage TAG $[16,17]$.

Undoubtedly, the production of high levels of EFA in engineered oilseeds will need the introduction of other genes involved in EFA metabolism, especially the specialized enzymes as-yet-unidentified from high-EFA accumulators. To facilitate this effort, we analyzed the FA compositions from developing $V$. galamensis seeds and generated the first assembly of $V$. galamensis transcriptome. As a result, nearly all genes involved in the processes of EFA metabolism, in particular, fatty acid modification, fatty acid $\beta$ oxidation, acyl editing, TAG storage, oil-body formation and transcription regulators were identified. Transient expression assays of some selected genes for enhancing EFA-enriched TAG accumulation in 
tobacco leaves were also performed. We further established a network for dissecting metabolic pathway involved in EFA and TAG biosynthesis based on the annotated transcriptome sequences and gene expression profiles. To the best of our knowledge, this study is the first report on analyzing the transcriptome data in oilseeds which produce EFA-enriched oils. The genes identified from $\mathrm{V}$. galamensis seeds will provide valuable resources for reprogramming natural epoxy oil in current established oilseed crops.

\section{Methods}

\section{Plant materials and fatty acid analysis}

Seeds of $V$. galamensis were obtained from National Infrastructure for Crop Germplasm Resources. In this study, $V$. galamensis plants were grown under normal growth condition at the experimental station of Shanxi Agricultural University, Taigu, China. Leaf, stem, and root tissues were harvested from five-weekold plants. Flowers were collected at the full bloom stage, and the sampling time was recorded as 0 days after pollination (0 DAP). Developing seed samples were harvested at 10, 17, 24, 31, 38, 45, and 52 DAP, respectively. All samples were frozen in liquid nitrogen, and stored at $-80^{\circ} \mathrm{C}$ until further use. The extraction of seed oil was performed as described in our previous study [18]. Fatty acid methyl esters (FAMEs) were prepared from total lipid extracts by adding $0.5 \mathrm{~mL}$ of sodium methoxide, with incubating for $30 \mathrm{~min}$. The methylation was quenched by the addition of $0.5 \mathrm{~mL}$ isooctane, the resulting FAMEs extract was measured by Gas Chromatography (Agilent 7890B).

\section{RNA extraction, library construction and sequencing}

For transcriptome sequencing, a mixed sample (T01) collected equally from different tissues was prepared. Three representative stages of $V$. galamensis seed development at 17 DAP (T02), 38 DAP (T03), and 45 DAP (T04) were also selected for cDNA library construction. Total RNA from each sample was isolated using Trizol Reagent (Shenggong, China) following the manufacturer's protocol. After checking the quality and quantity with NanoDrop spectrophotometer (NanoDrop Technologies, USA) and Agilent 2100 Bioanalyzer (Agilent Technologies, USA), mRNA was enriched using oligo (dT) beads and then broken into short pieces by random shearing. We further used these fragments as templates for first and second strand cDNA synthesis. Obtained cDNA fragments were then processed with end repair, Illumina's paired-end adapter ligation, size range selection, purification and PCR amplification. Finally, 12 paired-end cDNA libraries with three biological replicates in each indicated sample were constructed and then sequenced on flow cell using Illumina HiSeq ${ }^{\mathrm{TM}} 2500$ system in Biomarker Technology Corporation (Beijing, China).

\section{Transcriptome assembly}

After sequencing, RNA-Seq data were generated in the form of Fastq. The raw data were then processed by removing the reads with more than $5 \%$ of unknown nucleotides, the reads containing more than $20 \%$ of low-quality bases (quality score less than 30 ), empty reads and adaptor sequences. The filtered reads 
from all samples were then merged and de novo assembled using Trinity assembler with default parameters and 'K-mer size $=25^{\prime}$ [19]. Trinity program included three independent processing modules. Firstly, clean reads were extended into longer contigs by merging overlapping regions. Secondly, contigs from the same transcript were further connected based on paired-end match information. Finally, the sequences which could not be extended on either end were screened by redundancy removal tool. The unique consensus sequences were defined as unigenes, which represented the reference dataset for $V$. galamensis transcriptome.

\section{Sequence annotation and estimation of differentially expressed genes}

Gene functions were annotated by BLASTX searches (E-value < 1E-5) against public databases, including NCBI non-redundant (Nr), Swiss-Prot, Kyoto Encyclopedia of Genes and Genomes (KEGG), COG and KOG. Gene ontology (GO) terms were assigned based on Nr annotations using Blast2GO program, and $\mathrm{GO}$ tree was illustrated by WEGO tool [20]. The expression abundance of each unigene was counted and normalized using FPKM method, which could eliminate the effects of gene length and sequencing depth [21]. The quantitative FPKM values could be directly used to calculate the transcriptional difference between different samples. Differentially expressed genes (DEGs) were determined using the Bioconductor package (EBSeq), in which Benjamini-Hochberg model was selected to adjust for multiple tests. The threshold of DEG was defined with statistically significant false discovery rate (FDR) of at most 0.001 and fold change of at least 2 [22]. Functional enrichment analyses of DEGs in GO terms were performed using Fisher's exact test. Hyper geometric test was applied to determine DEGs significantly enriched in KEGG pathway [23].

\section{Transient expression of the target genes in Nicotiana benthamiana}

Each candidate gene was cloned in the plant expression vector pCAMBIA1303 and transformed into AGL1 strain of Agrobacterium tumefaciens. Transient overexpression of the selected genes individually or in combination in $N$. benthamiana leaf tissues was carried out as described in our previous study [18]. For coexpression assay, $A$. tumefaciens cultures containing the gene coding for $V$. galamensis epoxygenase and other target genes were mixed until the final OD600 of 0.25 prior to infiltration. The empty-vector infected leaves were used as blank controls.

\section{Quantitative real-time PCR Analysis}

The quantitative Real-Time PCR (qRT-PCR) was carried out in CFX96 Real-Time PCR system (Bio-Rad, USA) with three replicates. Following reaction conditions were applied: $95^{\circ} \mathrm{C}$ for $10 \mathrm{~min}, 40$ cycles of 95 ${ }^{\circ} \mathrm{C}$ for $15 \mathrm{~s}, 60^{\circ} \mathrm{C}$ for $1 \mathrm{~min}, 72^{\circ} \mathrm{C}$ for $20 \mathrm{~s}$. The melting curve was analyzed after the final cycle, ramping from $65^{\circ} \mathrm{C}$ to $95^{\circ} \mathrm{C}$ with increment of $0.5^{\circ} \mathrm{C} / 5 \mathrm{~s}$. The normalized relative expression was calculated using 
$2^{-\Delta \Delta \mathrm{Ct}}$ method. $V$. galamensis actin gene was employed as internal reference. Gene-specific primers were listed in Additional file 1.

\section{Results}

\section{Dynamic patterns of oil content and composition in developing $\mathrm{V}$. galamensis seeds}

To select the optimal stages for transcriptome sequencing, the oil accumulation patterns during $V$. galamensis seed development were analyzed (Fig. 1). The results showed that only a little oil was produced in the developing seeds at 10 and 17 DAP ( $1.77 \%$ and $1.84 \%$, respectively), but a most noticeable increase in seed oil content occurred from 24 to 38 DAP. Thereafter, the growth rate of oil content changed gradually till 45 DAP when the maximum oil content (35.8\%) was reached (Fig. 1a). We further monitored the dynamic transition for fatty acid components in $V$. galamensis seed oils, including vernolic acid (EFA), palmitic acid (16:0), stearic acid (18:0), oleic acid (18:1), linoleic acid (18:2) and linolenic acid (18:3). On the whole, the percentage of EFA showed a similar increasing trend with total oil accumulation in developing $V$. galamensis seeds. Accompanying the accumulation of EFA, oleic acid and linoleic acid contents were largely decreased. Palmitic acid level gradually declined during seed development. Additionally, there was little change in stearic acid and linolenic acid which make up a lower relative proportion in developing seeds (Fig. 1b).

\section{Transcriptome sequencing and de novo assembly}

To identify candidate genes associated with EFA biosynthesis and metabolism, we selected a mixed sample (T01) and three seed developing stages at 17 (T02), 38 (T03), and 45 DAP (T04), which are consistent with the initial, fast and final stages of EFA accumulation, for transcriptome sequencing. 12 cDNA libraries considering three biological replicates at each sample generated around 265 million raw reads. After quality-control processing, a total of $77.6 \mathrm{~Gb}$ nucleotide bases were obtained. The average Q30 base ratio (sequencing quality value) and GC percentage for each library was 92.75 and $44.94 \%$, respectively (Table 1). These reads were then de novo assembled by Trinity toolkit, resulting in 67,114 unigenes with a mean length of $729.96 \mathrm{nt}$ (nucleotides) and N50 length of $1316 \mathrm{nt}$ (Table 2). The assembled unigenes mainly ranged from 200 to $2000 \mathrm{nt}$, and unigene numbers had a trend of gradual decrease as the length of unigene increased. Of the unigenes, 25,321 (37.73\%) sequences had a shorter length range between 200 to $300 \mathrm{nt}, 9132$ (13.61\%) transcripts were between 1000 to $2000 \mathrm{nt}$, and 5261 (7.84\%) sequences were longer than $2000 \mathrm{nt}$ in length (Fig. 2a). 
Table 1

Statistics of $V$. galamensis sequencing data

\begin{tabular}{|llllll|}
\hline Sample & Clean Reads & Nucleotide Number & GC Content (\%) & Q20 (\%) & Q30 (\%) \\
\hline T01-1 & $21,768,314$ & $6,525,399,658$ & 44.86 & 97.89 & 92.46 \\
\hline T01-2 & $21,699,383$ & $6,500,347,146$ & 44.44 & 97.65 & 92.56 \\
\hline T01-3 & $22,052,818$ & $6,593,770,720$ & 45.36 & 98.13 & 93.14 \\
\hline T02-1 & $21,719,871$ & $6,490,837,274$ & 45.61 & 97.94 & 92.52 \\
\hline T02-2 & $21,457,055$ & $6,420,821,818$ & 44.98 & 97.28 & 92.47 \\
\hline T02-3 & $21,545,320$ & $6,444,265,190$ & 44.84 & 97.69 & 92.83 \\
\hline T03-1 & $21,419,474$ & $6,413,206,670$ & 44.26 & 98 & 92.84 \\
\hline T03-2 & $20,896,090$ & $6,248,410,846$ & 45.18 & 97.95 & 92.61 \\
\hline T03-3 & $21,811,624$ & $6,521,416,014$ & 44.51 & 98.39 & 93.31 \\
\hline T04-1 & $21,760,574$ & $6,508,648,268$ & 45.18 & 97.95 & 92.61 \\
\hline T04-2 & $21,402,719$ & $6,408,190,056$ & 44.73 & 97.92 & 92.96 \\
\hline T04-3 & $21,805,679$ & $6,520,398,848$ & 45.29 & 97.61 & 92.67 \\
\hline
\end{tabular}

Table 2

Summary details of assembled sequences produced by Trinity

\begin{tabular}{|lll|}
\hline Length Range (nt) & Contigs & Unigenes \\
\hline $200-300$ & $4,572,572(98.91 \%)$ & $25,321(37.73 \%)$ \\
\hline $300-500$ & $20,277(0.44 \%)$ & $15,885(23.67 \%)$ \\
\hline $500-1000$ & $14,633(0.32 \%)$ & $11,515(17.16 \%)$ \\
\hline $1000-2000$ & $10,474(0.23 \%)$ & $9,132(13.61 \%)$ \\
\hline $2000+$ & $5,025(0.11 \%)$ & $5,261(7.84 \%)$ \\
\hline Total Number & $4,622,981$ & 67,114 \\
\hline Total Length & $235,579,452$ & $48,990,485$ \\
\hline N50 Length & 48 & 1,316 \\
\hline Mean Length & 50.96 & 729.96 \\
\hline
\end{tabular}

Functional annotation, classification and DEG analysis

Based on sequence similarity search, 35,680 (53.16\%) unigenes were functionally annotated in at least one public database, whereas the function of rest sequences $(31,434)$ remained unknown (Table 3$)$. The E-value distribution of BLASTX hits showed that $38 \%$ of unigenes shared strong homology to previously 
reported sequences (<1.0E-100) (Fig. 2b). Unigenes from V. galamensis had the most similarities with genes from Vitis vinifera (7981), followed by Solanum lycopersicum (4183) and Theobroma cacao (3088) (Fig. 2c). Overall, the longer sequences were more easily annotated with BLAST hits (Fig. 2d).

Table 3

Summary of functional annotations

\begin{tabular}{|llll|}
\hline Annotated Database & Annotated Unigene Number & $\mathbf{3 0 0 < = l e n g t h < 1 0 0 0}$ & length>=1000 \\
\hline COG_Annotation & 12043 & 3975 & 5884 \\
\hline GO_Annotation & 26882 & 10248 & 11191 \\
\hline KEGG_Annotation & 8371 & 3254 & 3363 \\
\hline KOG_Annotation & 20521 & 7701 & 8530 \\
\hline Pfam_Annotation & 23945 & 8570 & 11869 \\
\hline Swissprot_Annotation & 23667 & 9337 & 10131 \\
\hline nr_Annotation & 35308 & 14017 & 13682 \\
\hline All_Annotated & 35680 & 14156 & 13705 \\
\hline
\end{tabular}

Based on $\mathrm{Nr}$ annotation, a total of $26,882(40.05 \%)$ unigenes could be assigned to three main GO categories, including biological process (21,364 unigenes, $31.83 \%)$, molecular function $(21,031$ unigenes, $31.34 \%)$ and cellular component (17,749 unigenes, $26.45 \%)$. Within the biological process, metabolic process and cellular process represented the dominant GO terms. In category of cellular component, cell part and cell were the two top sub-categories. The terms related to binding and catalytic activity were most abundant in molecular function (Fig. 3a). Eukaryotic Orthologous Groups (KOG) analysis showed that the matched 28,896 unigenes were divided into 26 function classes, with the signal transduction mechanism (2406 unigenes) as the largest group (Fig. 3b). From KEGG system, 8371 unigenes were annotated to 118 metabolic pathways (Additional file 2). The most predominant pathways were ribosome (ko03010, 771), followed by oxidative phosphorylation (ko00190, 350) and protein processing in endoplasmic reticulum (ko04141, 317). Moreover, we also annotated several regulation networks of lipid accumulation, including fatty acid metabolism (ko00071, 101), biosynthesis of unsaturated fatty acids (ko01040, 77), glycerolipid metabolism (ko005610, 72) and alpha-linolenic acid metabolism (ko00592, 21).

In our study, FPKM value was used to quantify the expression level of each unigene. Gene expression levels were further analyzed by pairwise comparisons across the developmental stages (initial, fast and final phases of EFA accumulation) to identify differentially expressed genes (DEGs). Our criteria led to the identification of 2345 DEGs across three developing samples. There were 531, 464, and 121 genes that were differentially expressed specifically in the group of 38 DAP vs 17 DAP, 45 DAP vs 38 DAP, and 45 DAP vs 17 DAP, respectively (Fig. 4a). In the comparison of 38 DAP vs 17 DAP, there existed the largest number of DEGs (1629) and up-regulated genes (999). Only 441 DEGs were identified in 45 DAP vs 17 
DAP, including 138 down- and 303 up-regulated genes (Fig. 4b). The results showed that a more remarkable change in gene expression occurred in 38 DAP vs 17 DAP, as compared to 45 DAP vs 38 DAP. It was noteworthy that the number of up-regulated genes outnumbered the down-regulated genes in all comparison groups.

To explore the functional differences among DEGs in developing $V$. galamensis seeds, GO and KEGG enrichment analyses were conducted. Firstly, 651 annotated DEGs were assigned to 42 functional subgroups within three main GO categories, implying that these DEGs were functionally involved in diverse physiological processes (Fig. 5). Among these unigenes, the most enriched terms were those involved in regulatory activity for DNA replication, G2/M transition of mitotic cell cycle, and histone phosphorylation. Furthermore, many DEGs were related with the process of fatty acid hydrolysis, fatty acid alpha-oxidation and unsaturated fatty acid biosynthesis (Additional file 3). We highlighted that these enzymes may play critical roles in determining the fatty acid composition in $\mathrm{V}$. galamensis seeds. Further, 495 DEGs were mapped to 87 KEGG pathways (Additional file 3). After pathway enrichment, we noticed that abundant DEGs were included in fatty acid biosynthesis, biosynthesis of unsaturated fatty acid, glycerolipid metabolism, glycosphingolipid biosynthesis, and sphingolipid metabolism, which were closely related to lipid accumulation in $\mathrm{V}$. galamensis seed.

\section{Characterization of genes contributing to high EFA accumulation in V. galamensis seeds}

Although EFA is greatly enriched in $V$. galamensis seed oil, the molecular mechanism underlying its high accumulation is still unclear. The primary purpose of our study is to uncover crucial genes involved in the high accumulation of EFA, and we expect these genes would be used in genetic manipulation for commercial production of industrial-valued epoxy oil in traditional oilseed crops. Based on functional annotations, we identified 116 unigenes relevant to epoxy oil metabolism (Additional file 4), including the previously reported VgDGAT1 and VgDGAT2 [13], further showing that transcriptome analysis is a powerful tool for mining genes of interest. We then fully characterized all critical steps and enzymes involved in EFA-enriched oil biosynthetic pathway (Fig. 6).

It is generally known that FA synthesis begins with the transformation of acetyl-CoA to molonyl-CoA catalyzed by rate-limiting acetyl-CoA carboxylase (EC: 6.4.1.2, ACCase), an enzyme complex composed of four subunits: alpha-carboxyltransferase $(a-C T)$, beta-carboxyltransferase $(\beta-C T)$, biotin carboxyl carrier protein (BCCP) and biotin carboxylase (BC). We detected nine unigenes encoding ACCase subunits (one for $\mathrm{a}-\mathrm{CT}$, one for $\beta-\mathrm{CT}$, four for $\mathrm{BCCP}$, and three for $\mathrm{BC}$ protein, respectively). The expression of these genes exhibited a coordinated pattern, with the highest expression level observed at 38 DAP. Next, an acyl carrier protein (ACP) was covalently linked to malnonyl group by malonyl-CoA:ACP transacylase (EC: 2.3.1.39, MCAT) to form malonyl-ACP, an important substrate for following acyl chain elongation. During FA elongation process, butyryl-ACP was initially generated after the first condensation cycle. This reaction was completed within the participation of four successive enzymes: beta-ketoacyl-ACP synthase III (EC: 2.3.1.180, KAS III), NADPH-dependent beta-ketoacyl-ACP reductase (EC: 1.1.1.100, KAR), 3-HydroxyacylACP dehydratase (EC: 4.2.1.159, HAD) and enoyl-ACP reductase (EC: 1.3.1.9, EAR). Butyryl-ACP was 
further elongated to C16-ACP during the following six cyclic reactions, each of which used the same enzymes as the former but KAS III was replaced by KAS I (EC: 2.3.1.41). Whereas the last elongation of C16-ACP to C18-ACP required a third condensing enzyme (KAS II, EC: 2.3.1.179). We detected nearly all unigenes involved in the biosynthesis of C18-ACP, but MCAT was missed in our data, which may be due to its rare expression abundance in $V$. galamensis seed. Considering malonyl-ACP produced from MCAT was an important substrate for following acyl chain elongation, it is possible that other unknown genes may determine the transfer of malonyl moiety to ACP. Most 18:0-ACP obtained from final elongation process could be desaturated by stearoyl-ACP desaturase (EC: 1.14.19.2, SAD). There are seven SAD family genes in Arabidopsis, and AtFAB2 (At2g43710) has a primary role in dehydrogenation reaction. We found that there existed only one SAD gene (homolog of AtFAB2) in $\mathrm{V}$. galamensis seed, with the highest expression at 38 DAP. The elongation of FA was terminated when acyl group was released by acyl-ACP thioesterase (EC: 3.1.2.14, Fat), yielding free FA molecular. Two Fat gene families, FatA with a preference for unsaturated 18:1-ACP and FatB showing marked activity on saturated FAs, were detected with 1 and 4 unigenes, respectively. The expression levels of FatA and FatB exhibited opposing trends, and only FatA expression was the highest at 38 DAP. These free FAs were ultimately linked to CoA by long chain acylCoA synthetase (EC: 6.2.1.3, LACS) and exported to endoplasmic reticulum (ER) for modification. In our dataset, 22 unigenes encoding LACS were successfully annotated. Two unigenes, one for ER-localized LACS8 and one for plastid-localized LACS9, were deemed as DEGs throughout seed development.

In ER, most acyl-CoAs needed to be transferred to PC pools via lysophosphatidylcholine acyltransferase (EC: 2.3.1.23, LPCAT) for further desaturation or modification. This reaction and its reversible reacylation catalyzed by LPCAT was the key component of acyl editing. Generally, oleic acid (C18:1) was channeled into PC, and then sequentially desaturated by ER-associated FAD2 and FAD3 to form C18:2 and C18:3. $\mathrm{V}$. galamensis seed contained $80 \%$ EFA, and this modified fatty acid was controlled by a divergent form of FAD2. In our transcriptome, we detected four unigenes encoding FAD2 desaturase. These genes were expressed at a relatively steady level, and quickly dropped at the final stage sampled, 45 DAP. We also discovered a novel FAD2 variant, which was designated VgFAD2-like. The FAD2-like enzyme was most closely related to delta 12 fatty acid epoxygenase identified in S. laevis (Fig. 7a), and this enzyme contained the typical histidine box domains (Fig. 7b). In addition, VgFAD2-like gene showed bell-shaped expression pattern with a peak at 38 DAP. The increased expression of this gene is coincided with the increased accumulation of EFA-containing TAG during $V$. galamensis seed development, suggesting that VgFAD2-like was likely a fatty acid epoxygenase in $V$. galamensis. FAD3 genes were detected with two copies, and their expressions were very low, especially when seeds progressed to mid- and late-stages. After modification, PC releases FA into acyl-CoA pool through a constant deacylation reaction by LPCAT. Alternatively, PC can remove its phosphocholine head group and then degrade into DAG by phosphatidylcholine:diacylglycerol cholinephosphotransferase (PDCT). Phospholipid:diacylglycerol acyltransferase (PDAT) can also directly transport FA from PC into the sn-3 position of DAG to produce TAG, which is referred to as PC-mediated TAG assembly (Fig. 6). The resulted FAs on CoA (including de novo synthesized, desaturated and modified FAs) were subsequently assembled into glycerol-3phosphate (G3P) to form TAG, referred to as the Kennedy pathway. In acyl editing pathway, we isolated 
one unigene of LPCAT1 and two unigenes of LPCAT2, and only LPCAT1 gene was highly expressed throughout all developmental stages. For Kennedy pathway-related enzymes, we found that there were four unigenes encoding GPAT, five encoding LPAT, three encoding PAP and four encoding DGAT (including two DGAT1 and two DGAT2). Among 16 unigenes in this pathway, 9 unigenes exhibited a high transcript level in $V$. galamensis seed, and five unigenes substantially up-regulated their expression level, including two GPAT genes, one DGAT1 and two DGAT2 at 38 DAP. For PC-mediated TAG synthesis, PDAT1 was ubiquitously expressed in developing seeds at all stages, while PDAT2 showed a linear rise during seed development up to $38 \mathrm{DAP}$, and then decreased to a low level at 45 DAP. However, the expression level of PDCT was negligible in V. galamensis seed.

Once synthesized, TAG is protected in the form of oil bodies which is stabilized by phospholipidcontaining proteins, such as oleosins, caleosins and steroleosins. We identified eight unigenes encoding oleosin, four encoding caleosin and two encoding steroleosin. In V. galamensis seeds, expression levels of oleosin genes were the most abundant, followed by caleosins and steroleosins. It is generally known that fatty acid $\beta$-oxidation is essential for catabolism of stored TAG in seed-oil storing plants. The suppression of the TAG-disassembling genes might be conducive to oil accumulation in oilseeds. Unexpectedly, we noticed the expression levels of multiple genes related to fatty acid $\beta$-oxidation, including acyl-CoA oxidase (ACX), enoyl-CoA hydratase (ECH), and 3-ketoacyl-CoA thiolase (KAT) displayed a significant up-regulation from 17 DAP to 38 DAP (Additional file 5). Finally, we examined the transcriptional profiles of certain transcription factors with potential roles in oil accumulation.

WRINKLED1 (WRI1) is a master regulator of plant oil synthesis. This TF belongs to the Apetala2 ethylene response element binding factor (AP2/EREB) family. We found that $V g W R / 1$ was quickly up-regulated in the early period of oil accumulation, then kept a relatively high expression in middle period, and finally declined in the late developing period in $V$. galamensis seed. These results supported a critical role for VgWRI 1 in the early and middle period of $V$. galamensis oil accumulation. FUSCA3 (FUS3) and ABSCISIC ACID INSENSITIVE4 (ABI4), two other lipid-metabolism-related transcription factors, were highly

expressed in the late period of oil accumulation in $V$. galamensis seeds. Except for these unigenes, we did not identify the transcripts for other TFs relevant to lipid metabolism, such as LEAFY COTYLEDON1 (LEC1), LEC2, and MYB89.

\section{Transient expression of selected genes for enhancing EFA-enriched TAG accumulation in tobacco leaves}

To determine whether the VgFAD2-like gene isolated from our transcriptome data has catalytic specificity for EFA formation, we transiently expressed this gene in tobacco leaves mediated by Agrobacterium infiltration. As expected, the presence of a large amount of EFA (8.2\%) was detected in the GC trace, suggesting the potential role of VgFAD2-like enzyme in manipulation of high-valued expoy oil (Table 4). Besides, we also tested the effect of VgLPCAT1 and VgPDCT on EFA incorporation in TAG. Using tobacco transient assay system, coexpression of VgLPCAT1 and VgFAD2-like gene resulted in further increased amounts of EFA compared to the individual expression of VgFAD2-like gene alone (Table 4). By contrast, there was no significant change in fatty acid composition for additional expression of $V g P D C T$ gene compared to the VgFAD2-like alone (Table 4). These observations indicated that VgLPCAT, rather than 
VgPDCT, contributed to more EFA accumulation in the TAG, possibly due to the increased efficiency of EFA removal from PC. In addition, the above-mentioned gene sets represent a likely promising resource for future metabolic engineering of EFA production in commercial oilseed crops like soybean.

Table 4

Fatty acid composition of Nicotiana benthamiana leaves transiently expressing the selected genes

\begin{tabular}{|llllllll|}
\hline Lines & \multicolumn{7}{l|}{ Fatty acid composition (\% of total) } \\
& $\mathbf{1 6 : 0}$ & $\mathbf{1 8 : 0}$ & $\mathbf{1 8 : 1}$ & $\mathbf{1 8 : 2}$ & $\mathbf{1 8 : 3}$ & $\mathbf{2 0 : 0}$ & EFA \\
\hline Control & $21.6 \pm$ & $7.3 \pm$ & $3.9 \pm$ & $16.4 \pm$ & $42.7 \pm$ & $7.1 \pm$ & 0 \\
& 0.3 & 0.1 & 0.1 & 0.3 & 0.7 & 0.2 & \\
\hline VgFAD2-like & $21.5 \pm$ & $7.9 \pm$ & $4.1 \pm$ & $17.5 \pm$ & $33.2 \pm$ & $5.8 \pm$ & $8.6 \pm 0.2$ \\
& 0.5 & 0.3 & 0.1 & 0.5 & 0.9 & 0.1 & \\
\hline VgFAD2-like + & $22.3 \pm$ & $7.6 \pm$ & $3.7 \pm$ & $17.9 \pm$ & $32.4 \pm$ & $6.4 \pm$ & $8.2 \pm 0.1$ \\
VgPDCT & 0.5 & 0.1 & 0.4 & 0.6 & 1.2 & 0.5 & \\
VgFAD2-like + & $21.4 \pm$ & $7.9 \pm$ & $4.3 \pm$ & $21.3 \pm$ & $22.5 \pm$ & $4 \pm 0.3$ & $19.4 \pm$ \\
VgLPCAT1 & 1.6 & 0.9 & 0.2 & 0.9 & 1.9 & & 0.4 \\
\hline Error bars $\pm \mathrm{SD}, \mathrm{n}=3$. & & & & & & & \\
\hline
\end{tabular}

\section{Validation of gene expression using quantitative real-time PCR}

To validate the RNA-seq data, twelve genes (a-CT, FatB, SAD, FAD2, FAD2-like, FAD3, GPAT, LPCAT, PDAT, WRI1, Oleosin, ACX) associated with lipid biosynthesis were analyzed by qRT-PCR. The results showed that the gene expression levels detected by qRT-PCR analysis are consistent with those obtained by transcriptome data (Fig. 8, Additional file 6), thus confirming that the data from RNA-Seq were reliable.

\section{Discussion}

Epoxy fatty acid (EFA) is an important unusual fatty acid with broad industrial uses. In nature, only a limited number of wild plants have the capacity to synthesize EFA in their seeds. However, the molecular mechanism of EFA accumulation in these plants is yet to be explored. Due to the high EFA percentage in seed oil, $V$. galamensis has been a model type for exploring the molecular mechanism and regulatory of EFA synthesis. Given the commercialization of EFA is limited by its unfavorable agronomy status, the analysis of EFA metabolism in $V$. galamensis seed will provide potential genetic resources for producing EFA in other plants via genetic engineering.

With the focus on effective and reliable data, RNA-seq has been widely applied in the identification of target genes at whole-transcriptome level, especially in non-model species, including some oilseed crops for which reference genomes are not available [24]. By the aid of transcriptome analysis, Tian et al successfully identified a list of important genes involved in unusual hydroxy fatty acid metabolism in Hiptage benghalensis seeds [25], which also verified the feasibility of our experiment design. In this study, for better understanding the EFA biosynthesis and regulation at the molecular level, transcriptome 
sequence was utilized for the identification of candidate genes contributing to high EFA accumulation and their expression profiles in developing $V$. galamensis seeds. Firstly, changes in oil content and FA profiles during seed development were recorded. Like the reports that oil accumulation often occurred at the mid-late stage of seed development in oilseed plants [23], the lipid in V. galamensis seed rapidly accumulated between 31 and 38 DAP, reaching a peak at 45 DAP and then decreasing. At 52 DAP, the seed was mature and tended to stop growing. Intriguingly, the trend of EFA accumulation was in parallel with lipid content, and this result was consistent with that of the study reported by Li et al [26]. Therefore, we selected three development stages of seed samples, which represented the initial, fast and final phases of EFA accumulation, respectively, for transcriptome analysis. To uncover more genes which are not exclusively expressed in developing seeds, we also sequenced a sample from RNA pool of different tissues. As a result, an assembly of 67,114 unigenes with a mean length of $729.96 \mathrm{nt}$ was generated for $V$. galamensis, which were comparable to transcriptome data from other oilseed plants, such as bitter melon [27], Malania oleifera [28], and Camelina sativa [29]. However, only 53.16\% of unigenes were functionally annotated, this relatively low rate might be due to the limited genomic information available for $V$. galamensis. In addition, non-annotated unigenes may be the consequence of having many shorter sequences, incomplete conserved domains or species-specific sequences.

Using linoleic acid (18:2)-PC as substrate, EFA was directly produced by FAD2-like enzyme (epoxygenase) at the $s n-2$ position of PC [13]. The linoleic acid content was high (over 38\%) at initial seed development until 17 DAP, and this quantity of linoleic acid seemed to be stored as ample substrate for further introduction of epoxy group. Except for EFA, linolenic acid (18:3) was also synthesized from linoleic acid via the desaturation catalyzed by FAD3 [30]. However, we observed that the linolenic acid content always remained at a relatively low level, and EFA was rapidly synthesized from 17 to 38 DAP. Accompanying the accumulation of EFA, transcript level of epoxygenase gene also increased steadily. Whereas, the expression of $F A D 3$ genes kept low levels throughout seed development. Low activity of $F A D 3$ seemed to determine the production of EFA by blocking the flow of linoleic acid substrate. These collective data may account for the relatively high content of EFA in developing V. galamensis seed.

In plants, most free fatty acids need to be assembled into storage TAG. Only a small fraction can be bound to membrane. V. galamensis seed contained over 70\% EFA, which was synthesized by $\Delta 12$ epoxygenase activity on linoleic acid bound to PC. Despite its main synthesis on PC, few EFA kept binding to PC in cells, but most of EFA was found to be stored in TAG during the maturation of $V$. galamensis seed. However, unusual fatty acids including EFA in the engineered plant seeds were mostly esterified to PC (membrane lipids), where they had limited reserves in TAG [11]. These facts implied that there should exist an active channel from its synthesis on PC to its accumulation on TAG in $V$. galamensis seeds. It is generally accepted that there are three pathways involved in the flux of newly generated FA from PC to TAG (Fig. 6). First, PC releases FA into acyl-CoA pool through a constant deacylation reaction by LPCAT, thus the modified FA could enter Kennedy pathway for TAG assembly. Second, PC removes phosphocholine head group and degrades into DAG by PDCT, the resulted DAG could be further utilized by DGAT to form TAG. Third, PC directly transfers FA into the sn-3 position of 
DAG to produce TAG by PDAT. To study the flux of EFA from PC to TAG, the above-mentioned lipid biosynthetic enzymes are of particular interest.

LPCAT enzymes play major roles in acyl editing, which include the incorporation of newly generated FA into PC (forward reaction), or transferring FA generated from PC to acyl-CoA pool through reversible acylation. Previous work has observed that the mutation of LPCAT genes in Arabidopsis resulted in a decrease of polyunsaturated fatty acid level [31]. In the case of unusual fatty acid, Lager et al first studied the substrate specificity and selectivity of LPCAT enzyme in its reversible reaction [32]. By biochemical method, they found LPCAT enzymes from the hydroxy fatty acid-accumulating plants catalyzed the acylation $s n-2$ position of PC with great preference for hydroxy group, while LPCAT enzymes from Arabidopsis did not exhibit such selectivity. These results suggested an important role for LPCAT enzyme in removing desaturated and modified fatty acid from PC to acyl-CoA pool. Arabidopsis genome contained two LPCAT genes, LPCAT1 (At1g12640) and LPCAT2 (At1g63050). We have identified one unigene of VgLPCAT1 (c9083.graph_c0) and two VgLPCAT2 unigenes (c33748.graph_c0, and c43652.graph_c0) in our transcriptome data, but little expression of VgLPCAT2 genes were detected. $V g L P C A T 1$ showed high expression during seed development, suggesting its roles in enriching acyl-CoA pool with EFA-CoA. Previous studies showed that PDCT channels PC-derived DAG to TAG in some oilseeds. Overexpression of castor bean PDCT gene enhanced novel product levels in hydroxy fatty acidproducing Arabidopsis seeds [15]. PDCT also regulated the polyunsaturated fatty acid accumulation in Arabidopsis [33]. However, the expression level of PDCT genes was nearly non-detectable in $V$. galamensis seeds. Similar to the case reported here, not any transcripts for PDCT were detected in transcriptomic analysis of bitter melon seeds, which produce high levels of eleostearic acid, an unusual conjugated fatty acid [27]. These suggested that PDCT genes might play a minor role in unusual fatty acid accumulation, at least for EFA and conjugated fatty acid. In the subsequent tobacco leaf transient assay, VgLPCAT1 and VgPDCT showed distinctive roles in the selective accumulation of EFA, which was consistent with their different expression patterns.

As important enzymes catalyzing the final step in TAG formation, both $V g D G A T$ and $V g P D A T$ genes had high expression levels in $V$. galamensis seed and thus appeared to contribute to EFA accumulation. In plants, these two types of enzymes are involved in the different routines. Generally, PDAT produces the TAG by transferring $s n-2$ FA from PC into $s n-3$ position of DAG, while DGAT converts fatty acid from acylCoA to the sn-3 position of diacylglycerol (DAG) in Kennedy pathway. In Arabidopsis, PDAT and DGAT1 were major enzymes with overlapping functions for catalyzing TAG production, while the function of DGAT2 remained unclear [34]. In plants accumulating unusual fatty acids, such as Ricinus communis and Vernicia fordii, DGAT2 genes were more highly expressed than DGAT1, thus DGAT2 was proposed to be important for incorporation of unusual fatty acids into TAG $[35,36]$. DGAT2 was also abundant in olive and oil palm for accumulating common fatty acids $[37,38]$. These results indicated that different plant species might have different routes to produce TAG. Although Yu et al have showed that VgDGATs have higher specificity for acyl substrates containing EFA [12], we found DGAT1, DGAT2 and PDAT all contributed to the EFA enrichment, with VgPDAT as the major player. In general, the high level of EFA 
incorporated into TAG molecules is usually dependent not only on the active synthesis of EFA, but on the efficient assembly mechanism for the incorporation of EFA into TAG.

Similar to the reports in other oilseeds, many oleosins, caleosins and steroleosins with very high transcripts were detected in V. galamensis seeds [39]. These proteins provided a central hydrophobic region, being capable of protecting TAG in the form of oil bodies. Considering the sequence variability of amphipathic domain in oleosins from different species, Yang et al suggested that oleosins may have some substrate specificity [39]. It is possible that one or more of oleosins from $\mathrm{V}$. galamensis may specifically package EFA-rich TAG to oil bodies. Notably, three unigenes (c22074.graph_c0, c27706.graph_c0 and c7651.graph_c0) encoding oleosin-like proteins were expressed in exceptional abundance at all three seed development stages. These proteins may be the major oleosin isoforms in oil-body formation of $V$. galamensis seed.

It was interesting that $\beta$-oxidation was active during $V$. galamensis seed development. This pathway was originally upregulated during seed germination, which is a main process to provide essential energy for seedling growth [40]. However, we found that several $\beta$-oxidation related genes were highly upregulated during EFA quickly accumulated stage. The activity of $\beta$-oxidation was quite distinct from that of FA and TAG biosynthesis stage in other oil seeds, except for the pattern in tung tree, which contained high levels of uncommon eleostearic fatty acids [41]. It is generally known that high concentrations of free unusual fatty acids could be toxic for cellular environment. From an evolutionary point of view, the native unusual fatty acid accumulators must have developed highly efficient mechanisms to package most unusual fatty acids into seed oil bodies, and they may also maintained active pathways for disposal of excess free unusual fatty acids [41]. Our observations regarding up-regulated $\beta$-oxidation pathway in the fast oil accumulation stage of developing seeds seem to be paradoxical since developing seeds are usually active in fatty acid and oil synthesis. However, $V$. galamensis seed might be evolutionarily programmed to activate the $\beta$-oxidation pathway to degrade excess free EFAs which are not incorporated into TAGs during rapid oil accumulation period.

Transcription factors (TFs) are key regulators in metabolic networks, in which one TF can simultaneously regulate the expression of multiple genes while one gene can be simultaneously regulated by multiple TFs [42]. In this study, we annotated many TFs, indicating that $V$. galamensis seed development is controlled by a complex of regulatory network. Previous studies indicated that WRI1, FUS3, and ABI4 were the key TFs controlling oil accumulation in plant seeds [43]. The analysis of $\mathrm{V}$. galamensis transcriptome showed that these three TFs had high expression levels. Overexpression of WR/1 significantly enhanced the seed oil content in transgenic plants. Increasing evidence has showed that WRI1 is a master regulator in controlling the gene expression of lipid genes in the pathway of fatty acid biosynthesis [44]. Notably, we found that WRI1 is important in the early and middle period of $V$. galamensis oil accumulation, as reported for Arabidopsis [45]. Studies have also revealed that both FUS3 and ABI4 are involved in direct or indirect regulation of the fatty acid biosynthesis and TAG accumulation in other plants $[46,47]$. These two TFs may promote oil accumulation in the late period of $V$. galamensis lipid synthesis. However, we did not detect the transcripts for other lipid-regulated TFs, such as LEC1, LEC2, and MYB89 in any stages 
tested, suggesting more efforts should be paid to enlarge the pool of transcription factors for this species in future studies.

\section{Conclusion}

V. galamensis seed is valued for its high content of natural EFA. Transcriptome sequencing from developing $V$. galamensis seeds and one mixed sample was carried out to identify candidate genes that may contribute to the accumulation of EFA-enriched oil. A total of 67,114 unigenes with an N50 length of 1,316 nt were assembled from 65 million raw cDNA reads, and $53.16 \%$ of them had homology with known genes. Focused on EFA accumulation, we have identified nearly all of the known genes for de novo EFA biosynthesis, exportation from the plastid, TAG assembly and storage, including Kennedy pathway and PC-mediated TAG synthesis, and TAG degradation. By analyzing the expression profiles, we found that the genes associated with acyl editing, fatty acid $\beta$-oxidation, triacylglycerol assembly and oil body formation had greater expression levels at the mid-development stage (38 DAP), which are consistent with the fast phase of EFA accumulation in $V$. galamensis seed, indicating their crucial roles in EFA production. The transient assay of selected genes resulted in a synergistic increase of EFA-enriched TAG accumulation in tobacco leaves. Moreover, we isolated some transcription factors (such as WRI1, FUS3 and ABI4) which putatively regulated the $V$. galamensis seed oils enriched in EFA. The sequence and gene expression data presented here will enrich our understanding of EFA biosynthesis and regulation in $V$. galamensis seed, providing essential information for commercial production of natural epoxy oil in the established oilseed crops by gene engineering.

\section{Abbreviations}

ACCase: Acetyl-CoA carboxylase; ACP: Acyl carrier protein; ACX: Acyl-CoA oxidase; BC: Biotin carboxylase; BCCP: Biotin carboxyl carrier protein; DAP: Days after pollination; DEG: Differentially expressed gene; DGAT: Acyl-CoA:diacylglycerol acyltransferase; EAR: Enoyl-ACP reductase; ECH: Enoyl-CoA hydratase; EFA: Epoxy fatty acid; FA: Fatty acid; FAD2: $\triangle 12$-fatty acid desaturase; FAD2-like: $\Delta 12$-fatty acid epoxygenase; FAD3: $\triangle 15$-fatty acid desaturase; FAME: Fatty acid methyl ester; Fat: Acyl-ACP thioesterase; FPKM: Fragments Per Kilobase of transcript per Million fragments mapped; FUS3: FUSCA3; GO: Gene ontology; HAD: 3-Hydroxyacyl-ACP dehydratase; KAR: NADPH-dependent beta-ketoacyl-ACP reductase; KAS: Beta-ketoacyl-ACP synthase; KAT: 3-ketoacyl-CoA thiolase; KOG: Eukaryotic Orthologous Groups; KOG: Kyoto Encyclopedia of Genes and Genomes; LACS: Long chain acyl-CoA synthetase; LEC1: LEAFY COTYLEDON 1; LPCAT: Lysophosphatidylcholine acyltransferase; MCAT: Malonyl-CoA:ACP transacylase; Nr: NCBI non-redundant database; PC: Phosphatidylcholine; PDAT: Phospholipid:diacylglycerol acyltransferase; PDCT: Phosphatidylcholine:diacylglycerol cholinephosphotransferase; SAD: StearoylACP desaturase; TAG: Triacylglycerol; a-CT: Alpha-carboxyltransferase; $\beta$-CT: Beta-carboxyltransferase.

\section{Declarations}

\section{Ethics approval and consent to participate}


Not applicable.

\section{Consent for publication}

Not applicable.

\section{Availability of data and material}

The datasets generated and/or analyzed during the current study are included in Additional Tables.

\section{Competing interests}

The authors declare that they have no competing interests.

\section{Fundings}

This work was supported by the National Natural Science Foundation of China (Grant No. 31401430), Key Research and Development Project of Shanxi Province (201703D221002-3), State Ministry of Agriculture "948" Project (2014-Z39), Shanxi Province Key Projects of Coal-based Science and Technology (FT-2014-01), Science and technology innovation of higher education of Shanxi province (2021L112), and Research Project Supported by Shanxi Scholarship Council of China (2015-064).

\section{Authors' contributions}

YS, XYJ and RZL conceived the original research plans. YS, BLL, JAX and XDW conducted the experiments and analyzed the data. HLC provided some experimental materials. YS, BLL, JAX and XDW performed the tobacco transient assay. YS, HLC, XYJ and drafted the manuscript. RZL revised the manuscript critically. All authors read and approved the manuscript.

\section{Acknowledgements}

Not applicable.

\section{Author details}

Institute of Molecular Agriculture and Bioenergy, Shanxi Agricultural University, Taigu, China

*Correspondence: rli2001@126.com

\section{References}

1. Dyer JM, Stymne S, Green AG, Carlsson AS. High-value oils from plants. Plant J. 2008;54(4):640655.

2. Kumar A, Sharma A, Upadhyaya KC. Vegetable oil: Nutritional and industrial perspective. Curr Genomics. 2016;17(3):230-240. 
3. Ncube I, Gitlesen T, Adlercreutz P, Read JS, Mattiasson B. Fatty acid selectivity of a lipase purified from Vernonia galamensis seed. Biochim. Biophys Acta. 1995;1257(2):149-156.

4. Carlson KD, Chang SP. Chemical epoxidation of a natural unsaturated epoxy seed oil from Vernonia galamensis and a look at epoxy oil markets. J Am Oil Chem Soc. 1985;62(5):934-939.

5. Cahoon EB, Ripp KG, Hall SE, Mcgonigle B. Transgenic production of epoxy fatty acids by expression of a cytochrome P450 enzyme from Euphorbia lagascae seed. Plant Physiol. 2002;128(2):615-624.

6. Perdue RE, Carlson KD, Gilbert MG. Vernonia galamensis, Potential new crop source of epoxy acid. Economic Botany 1986;40:54-68.7.

7. Baye T, Becker HC, Witzkeehbrecht SV. Vernonia galamensis, a natural source of epoxy oil: variation in fatty acid composition of seed and leaf lipids. Ind Crops Prod. 2005;21(2):257-261.

8. Thompson AE, Dierig DA, Kleiman R. Variation in Vernonia galamensis flowering characteristics, seed oil and vernolic acid contents. Ind Crops Prod. 1994;3(3):175-183.

9. Aznar-Moreno JA, Durrett TP. Review: Metabolic engineering of unusual lipids in the synthetic biology era. Plant Sci. 2017;263:126-131.

10. Hatanaka T, Shimizu R, Hildebrand D. Expression of a Stokesia laevis epoxygenase gene. Phytochemistry. 2004;65(15):2189-2196.

11. Singh S, Thomaeus S, Lee M, Stymne S, Green A. Transgenic expression of a delta 12-epoxygenase gene in Arabidopsis seeds inhibits accumulation of linoleic acid. Planta. 2001;212(5-6):872-879.

12. Yu K, Li R, Hatanaka T, Hildebrand D. Cloning and functional analysis of two type 1 diacylglycerol acyltransferases from Vernonia galamensis. Phytochemistry. 2008;69(5):1119-1127.

13. Li R, Yu K, Hatanaka T, Hildebrand DF. Vernonia DGATs increase accumulation of epoxy fatty acids in oil. Plant Biotechnol J. 2010;8(2):184-195.

14. Cahoon EB, Dietrich CR, Meyer K, Damude HG, Dyer JM, Kinney AJ. Conjugated fatty acids accumulate to high levels in phospholipids of metabolically engineered soybean and Arabidopsis seeds. Phytochemistry. 2006;67(12):1166-1176.

15. Hu Z, Ren Z, Lu C. The phosphatidylcholine diacylglycerol cholinephosphotransferase is required for efficient hydroxy fatty acid accumulation in transgenic Arabidopsis. Plant Physiol. 2012;158(4):1944-1954.

16. Bates PD, Browse J. The pathway of triacylglycerol synthesis through phosphatidylcholine in Arabidopsis produces a bottleneck for the accumulation of unusual fatty acids in transgenic seeds. Plant J. 2011;68(3):387-399.

17. Yu XH, Cahoon RE, Horn PJ, Shi H, Prakash RR, Cai Y, Hearney M, Chapman KD, Cahoon EB, Schwender $\mathrm{J}$ et al. Identification of bottlenecks in the accumulation of cyclic fatty acids in camelina seed oil. Plant Biotechnol J. 2018;16(4):926-938.

18. Ji XJ, Mao X, Hao QT, Liu BL, Xue JA, Li RZ. Splice variants of the castor WR/1 gene upregulate fatty acid and oil biosynthesis when expressed in tobacco leaves. Int J Mol Sci. 2018;19(1):146-157. 
19. Grabherr MG, Haas BJ, Yassour M, Levin JZ, Thompson DA, Amit I, Xian A, Lin F, Raychowdhury R, Zeng Q. Trinity: reconstructing a full-length transcriptome without a genome from RNA-Seq data. Nat Biotechnol. 2011;29(7):644-652.

20. Ye J, Fang L, Zheng H, Zhang Y, Chen J, Zhang Z, Wang J, Li S, Li R, Bolund L et al. WEGO: a web tool for plotting GO annotations. Nucleic Acids Res. 2006;34:W293-297.

21. Wesolowski S, Birtwistle MR, Rempala GA. A comparison of methods for RNA-Seq differential expression analysis and a new empirical Bayes approach. Biosensors. 2013;3(3):238-258.

22. Leng N, Dawson JA, Thomson JA, Ruotti V, Rissman Al, Smits BM, Haag JD, Gould MN, Stewart RM, Kendziorski C. EBSeq: An empirical Bayes hierarchical model for inference in RNA-seq experiments. Bioinformatics. 2013;29(8):1035.

23. Huang J, Hao X, Jin Y, Guo X, Shao Q, Kumar KS, Ahlawat YK, Harry DE, Joshi CP, Zheng Y. Temporal transcriptome profiling of developing seeds reveals a concerted gene regulation in relation to oil accumulation in Pongamia (Millettia pinnata). BMC Plant Biology. 2018;18(1):140-156.

24. Gongora-Castillo E, Buell CR. Bioinformatics challenges in de novo transcriptome assembly using short read sequences in the absence of a reference genome sequence. Nat Prod Rep. 2013;30(4):490-500.

25. Tian B, Lu T, Xu Y, Wang R, Chen G. Identification of genes associated with ricinoleic acid accumulation in Hiptage benghalensis via transcriptome analysis. Biotechnol Biofuels. 2019;12:1632.

26. Li R, Yu K, Hildebrand DF. DGAT1, DGAT2 and PDAT expression in seeds and other tissues of epoxy and hydroxy fatty acid accumulating plants. Lipids. 2010;45(2):145-157.

27. Yang P, Li X, Shipp MJ, Shockey JM, Cahoon EB. Mining the bitter melon (Momordica charantia I.) seed transcriptome by 454 analysis of non-normalized and normalized cDNA populations for conjugated fatty acid metabolism-related genes. BMC Plant Biology. 2010;10:250.

28. Yang T, Yu Q, Xu W, Li DZ, Chen F, Liu A. Transcriptome analysis reveals crucial genes involved in the biosynthesis of nervonic acid in woody Malania oleifera oilseeds. BMC Plant Biology. 2018;18(1):247.

29. Liang C, Liu X, Yiu SM, Lim BL. De novo assembly and characterization of Camelina sativa transcriptome by paired-end sequencing. BMC Genomics. 2013;14:146.

30. Yang Q, Fan C, Guo Z, Qin J, Wu J, Li Q, Fu T, Zhou Y. Identification of FAD2 and FAD3 genes in Brassica napus genome and development of allele-specific markers for high oleic and low linolenic acid contents. Theor Appl Genet. 2012;125(4):715-729.

31. Wang L, Kazachkov M, Shen W, Bai M, Wu H, Zou J. Deciphering the roles of Arabidopsis LPCAT and $\mathrm{PAH}$ in phosphatidylcholine homeostasis and pathway coordination for chloroplast lipid synthesis. Plant J. 2014;80(6):965-976.

32. Lager I, Yilmaz JL, Zhou XR, Jasieniecka K, Kazachkov M, Wang P, Zou J, Weselake R, Smith MA, Bayon S et al. Plant acyl-CoA:lysophosphatidylcholine acyltransferases (LPCATs) have different specificities in their forward and reverse reactions. J Biol Chem. 2013;288(52):36902-36914. 
33. Lu C, Xin Z, Ren Z, Miquel M, Browse J. An enzyme regulating triacylglycerol composition is encoded by the ROD1 gene of Arabidopsis. Proc Natl Acad Sci. 2009;106(44):18837-18842.

34. Peng H, Moghaddam L, Brinin A, Williams B, Mundree S, Haritos VS. Functional assessment of plant and microalgal lipid pathway genes in yeast to enhance microbial industrial oil production. Biotechnol Appl Biochem. 2018;65(2):138-144.

35. Burgal J, Shockey J, Lu C, Dyer J, Larson T, Graham I, Browse J. Metabolic engineering of hydroxy fatty acid production in plants: RcDGAT2 drives dramatic increases in ricinoleate levels in seed oil. Plant Biotechnol J. 2008;6(8):819-831.

36. Shockey JM, Gidda SK, Chapital DC, Kuan JC, Dhanoa PK, Bland JM, Rothstein SJ, Mullen RT, Dyer JM. Tung tree DGAT1 and DGAT2 have nonredundant functions in triacylglycerol biosynthesis and are localized to different subdomains of the endoplasmic reticulum. Plant Cell. 2006;18(9):22942313.

37. Banilas G, Karampelias M, Makariti I, Kourti A, Hatzopoulos P. The olive DGAT2 gene is developmentally regulated and shares overlapping but distinct expression patterns with DGAT1. Journal of Experimental Botany. 2011;62(2):521-532.

38. Jin Y, Yuan Y, Gao L, Sun R, Chen L, Li D, Zheng Y. Characterization and Functional analysis of a type 2 diacylglycerol acyltransferase (DGAT2) gene from oil palm (Elaeis guineensis Jacq.) mesocarp in Saccharomyces cerevisiae and transgenic Arabidopsis thaliana. Front Plant Sci. 2017;8:1791.

39. Yang Z, Ji H, Liu D. Oil biosynthesis in underground oil-rich storage vegetative tissue: Comparison of Cyperus esculentus tuber with oil seeds and fruits. Plant Cell Physiol. 2016;57(12):2519-2540.

40. Fulda M, Schnurr J, Abbadi A, Heinz E, Browse J. Peroxisomal Acyl-CoA synthetase activity is essential for seedling development in Arabidopsis thaliana. Plant Cell. 2004;16(2):394-405.

41. Cui P, Lin Q, Fang D, Zhang L, Li R, Cheng J, Gao F, Shockey J, Hu S, Lu S. Tung tree (Vernicia fordii, Hemsl.) genome and transcriptome sequencing reveals co-ordinate up-regulation of fatty acid betaoxidation and triacylglycerol biosynthesis pathways during eleostearic acid accumulation in seeds. Plant Cell Physiol. 2018;59(10):1990-2003.

42. Manan S, Chen B, She G, Wan X, Zhao J. Transport and transcriptional regulation of oil production in plants. Crit Rev Biotechnol. 2017;37(5):641-655.

43. Xiu Y, Wu G, Tang W, Peng Z, Bu X, Chao L, Yin X, Xiong J, Zhang H, Zhao X et al. Oil biosynthesis and transcriptome profiles in developing endosperm and oil characteristic analyses in Paeonia ostii var. lishizhenii. J Plant Physiol. 2018;228:121-133.

44. Pouvreau B, Baud S, Vernoud V, Morin V, Py C, Gendrot G, Pichon JP, Rouster J, Paul W, Rogowsky PM. Duplicate maize Wrinkled1 transcription factors activate target genes involved in seed oil biosynthesis. Plant Physiol. 2011;156(2):674-686.

45. Baud S, Wuilleme S, To A, Rochat C, Lepiniec L. Role of WRINKLED1 in the transcriptional regulation of glycolytic and fatty acid biosynthetic genes in Arabidopsis. Plant J. 2009;60(6):933-947.

46. Kim HU, Lee KR, Jung SJ, Shin HA, Go YS, Suh MC, Kim JB. Senescence-inducible LEC2 enhances triacylglycerol accumulation in leaves without negatively affecting plant growth. Plant Biotechnol J. 
2015;13(9):1346-1359.

47. S RV, Kumari P, Rupwate SD, Rajasekharan R, Srinivasan M. Exploring triacylglycerol biosynthetic pathway in developing seeds of chia (Salvia hispanica L.): A transcriptomic approach. PloS One. 2015;10(4):e0123580.

\section{Figures}

a

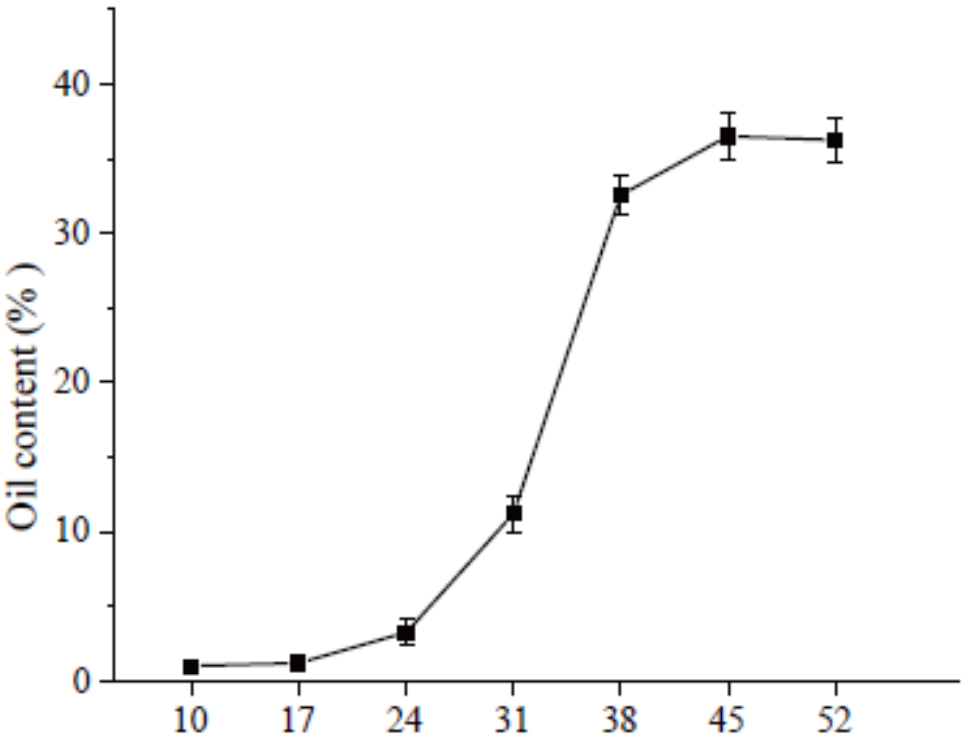

Days after pollination

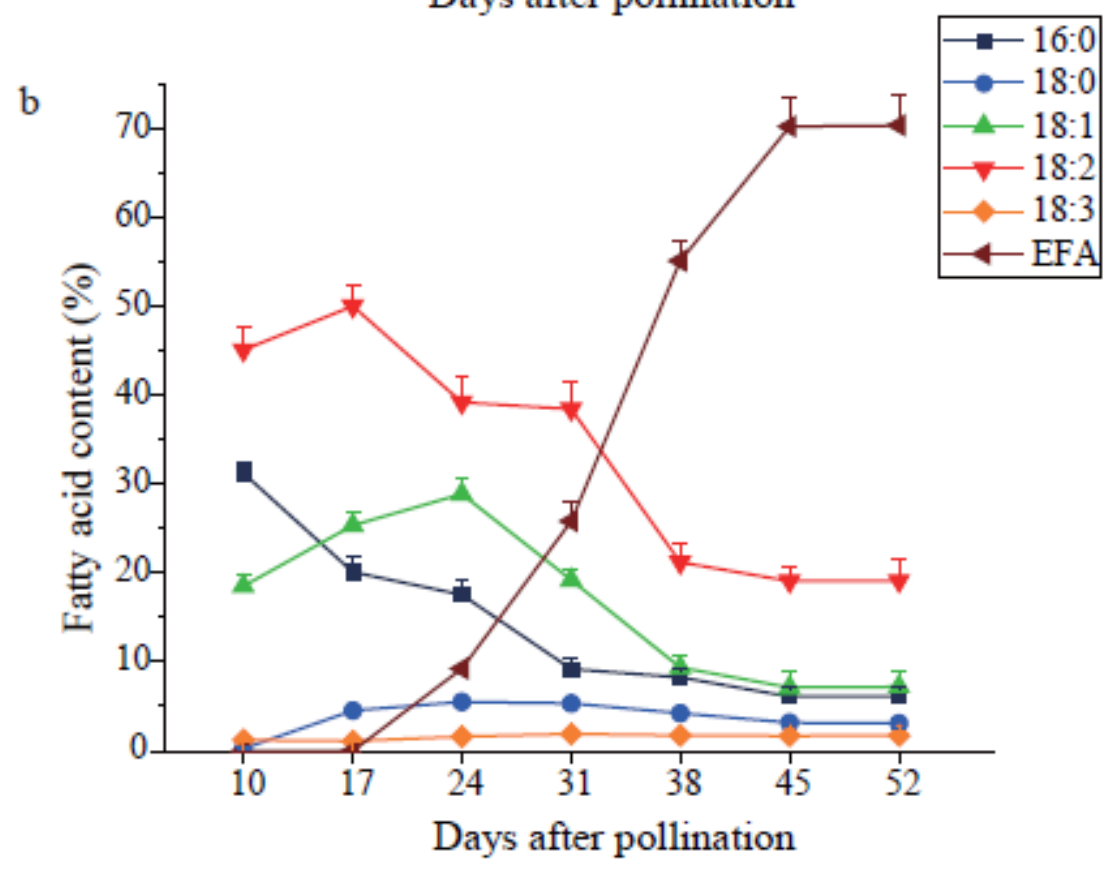

\section{Figure 1}

Lipid content and composition dynamics across the developmental stages of $\mathrm{V}$. galamensis seeds. (a) The lipid content of developing V. galamensis seeds. (b) The composition of the six major fatty acids in 
developing $V$. galamensis seeds (mean $\pm S D, n=3$ ). Seeds were harvested at 10 days after pollination (DAP, immature stage), and then every 7 days until 52 DAP (mature stage).

a

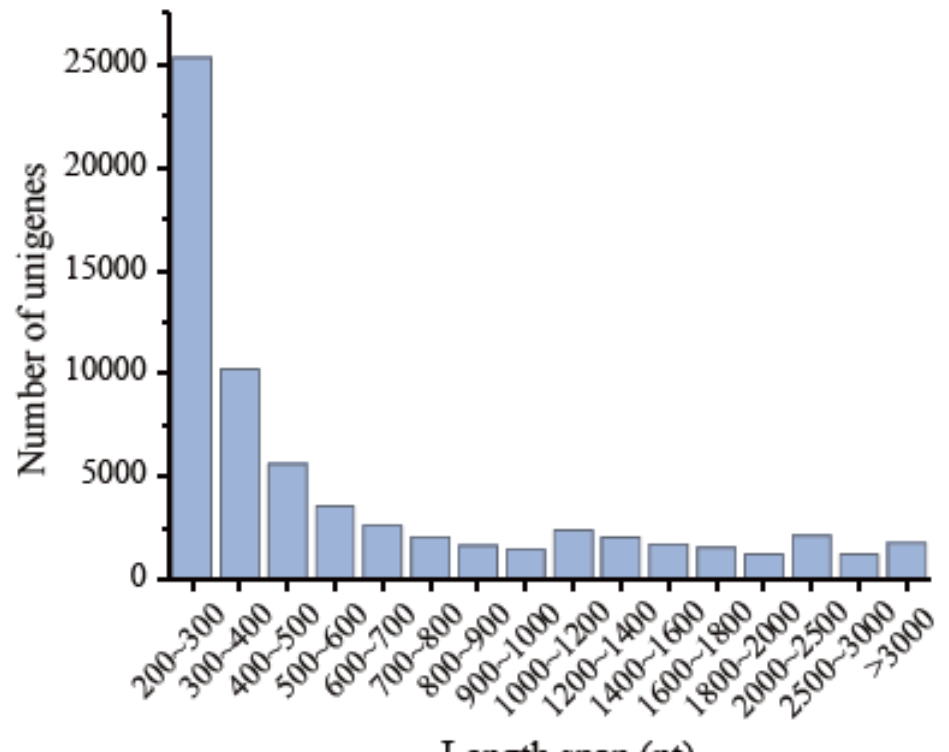

Length span (nt)

c

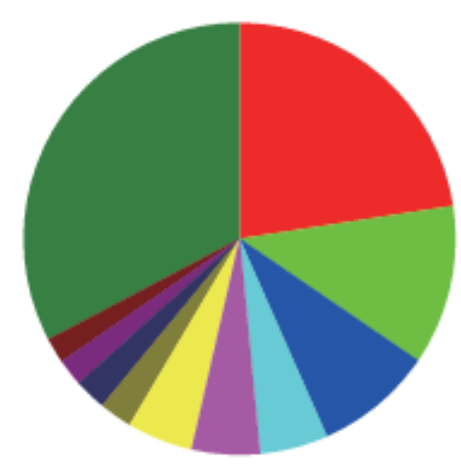

Species distribution $\mathrm{b}$

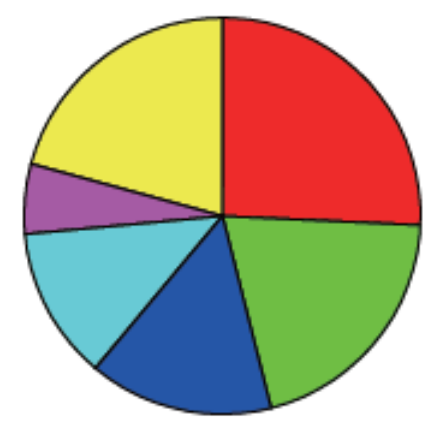

$1 \mathrm{E}-50$ to $1 \mathrm{E}-10$

$1 \mathrm{E}-100$ to $1 \mathrm{E}-50$

$1 \mathrm{E}-150$ to $1 \mathrm{E}-100$

0 to $1 \mathrm{~B}-150$

E-value distribution

d

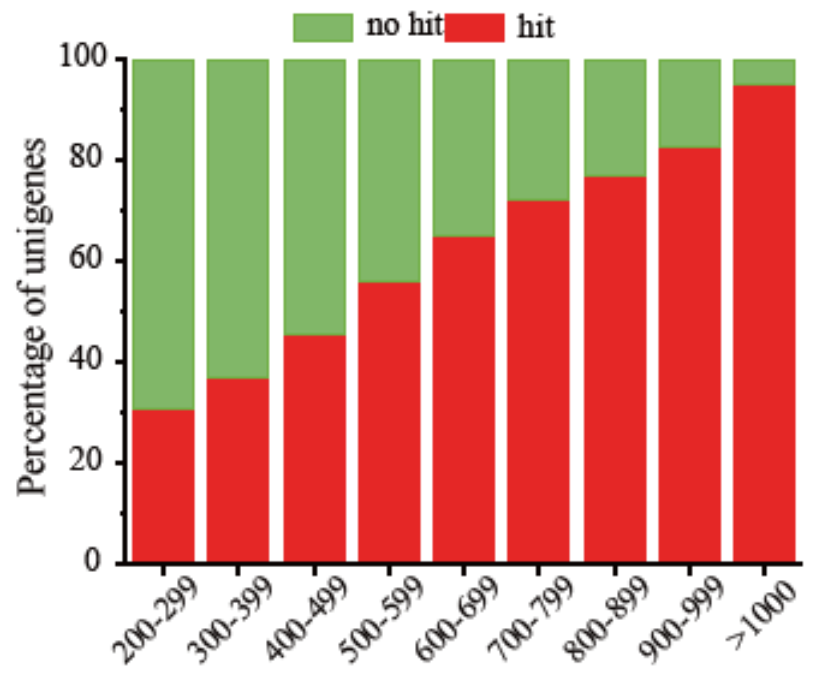

Length distribution of unigenes

\section{Figure 2}

Characteristics of unigenes in V. galamensis. (a) Size distribution of all assembled unigenes. (b) E-value distribution of best BLASTX hits for each unigene. (c) Species distribution of top BLAST hits for each unigene in $\mathrm{Nr}$ database. (d) Length of unigenes with hits or no hits. 

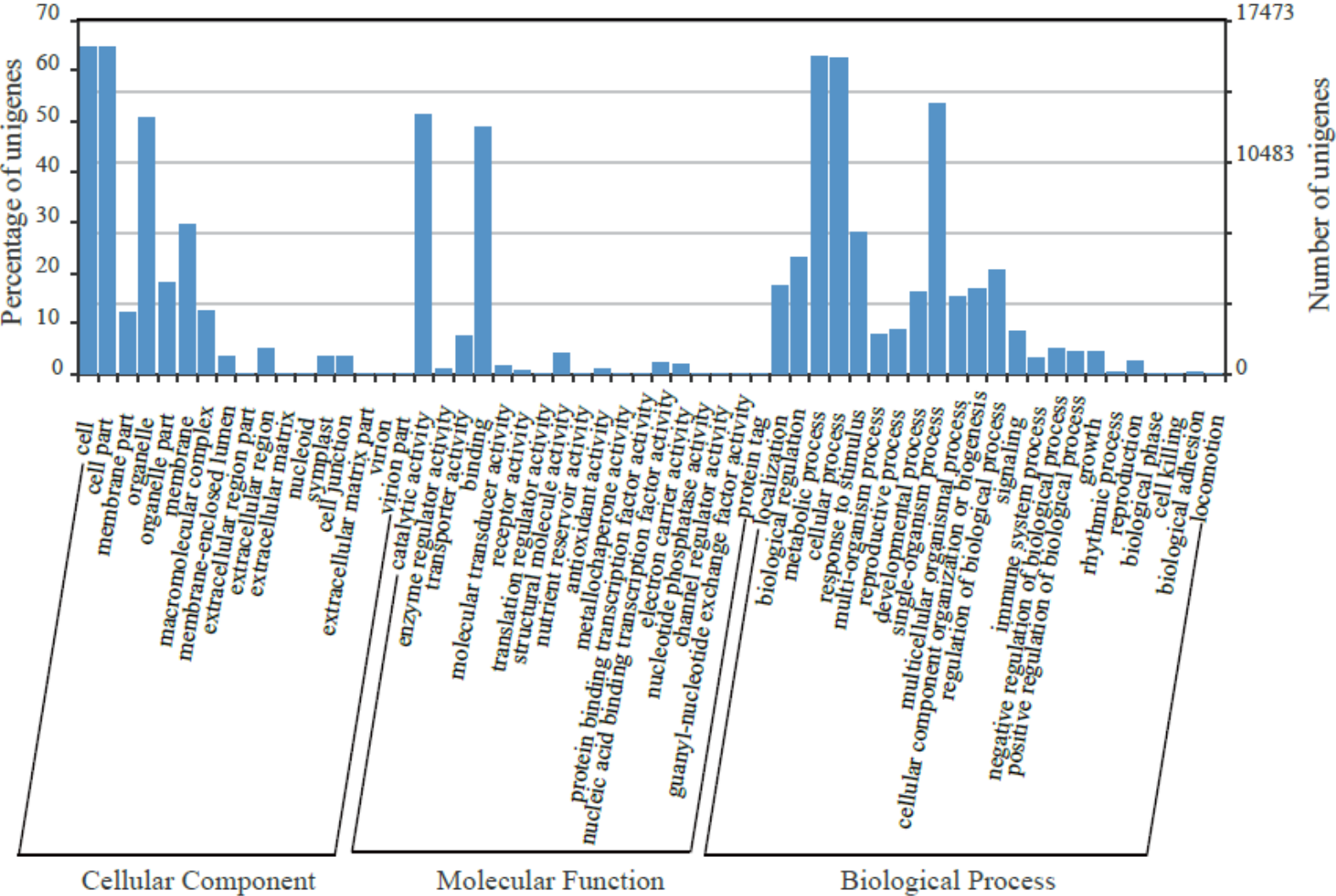

b

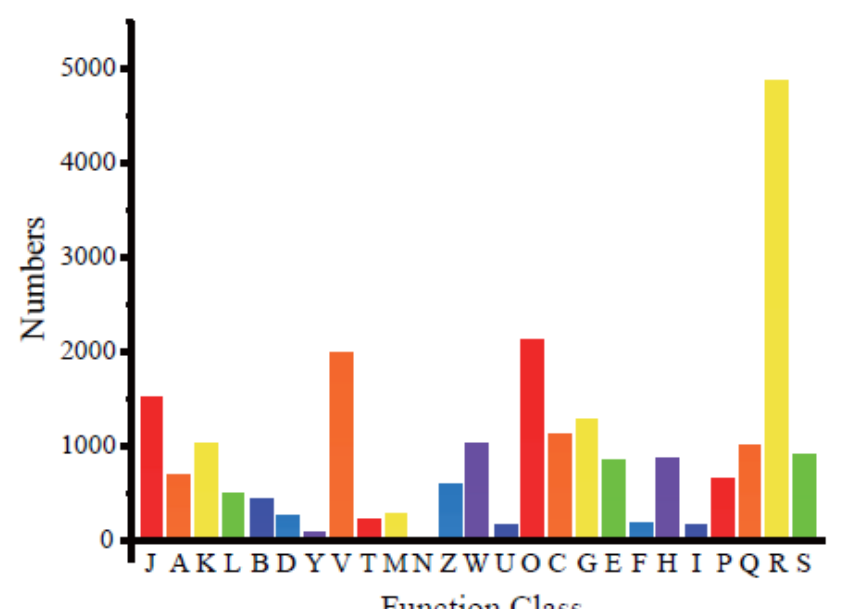

J Translation, ribosomal structure and biogenesis

$[A]$ RNA processing and modification

[K] Transcription

[L] Replication, recombination and repair

[B] Chromatin structure and dynamics

[D] Cell cycle control, cell division, chromosome partitioning

[Y] Nuclear structure

[V] Defense mechanisms

[T] Signal transduction mechanisms

[M] Cell wall/membrane/envelope biogenesis

[N] Cell motility

[Z] Cytoskeleton

[W] Extracellular structures

$[\mathrm{U}]$ Intracellular trafficking, secretion, and vesicular transport

[O] Posttranslational modification, protein turnover, chaperones

[C] Energy production and conversion

[G] Carbohydrate transport and metabolism

[E] Amino acid transport and metabolism

[F] Nucleotide transport and metabolism

[H] Coenzyme transport and metabolism

[1] Lipid transport and metabolism

$[\mathrm{P}]$ Inorganic ion transport and metabolism

[Q] Secondary metabolites biosynthesis, transport and catabolism

[R] General function prediction only

[S] Function unknown

\section{Figure 3}

Functional annotation and classification of unigenes in V. galamensis. (a) Overall classification of 21,364 unigenes distributed into major GO categories. (b) Eukaryotic Orthologous Groups (KOG) analysis of 28,896 unigenes which were classified into 26 functional groups. 


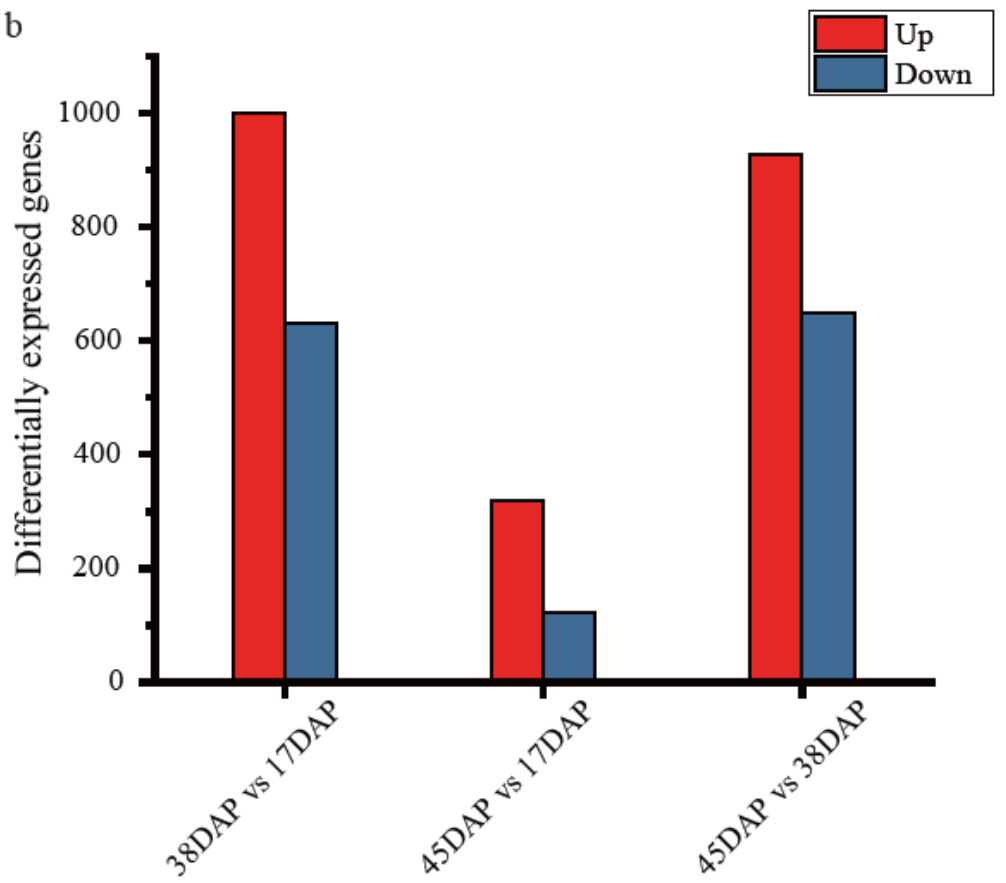

45DAP vs 38DAP

Comparison groups

\section{Figure 4}

Distribution of differentially expressed genes (DEGs) in V. galamensis at different developmental stages.

(a) Number of DEGs in different comparisons. (b) Venn diagram of DEGs in different comparisons: $38 D A P$ vs $17 D A P, 45 D A P$ vs 38DAP, and 45DAP vs 17DAP.
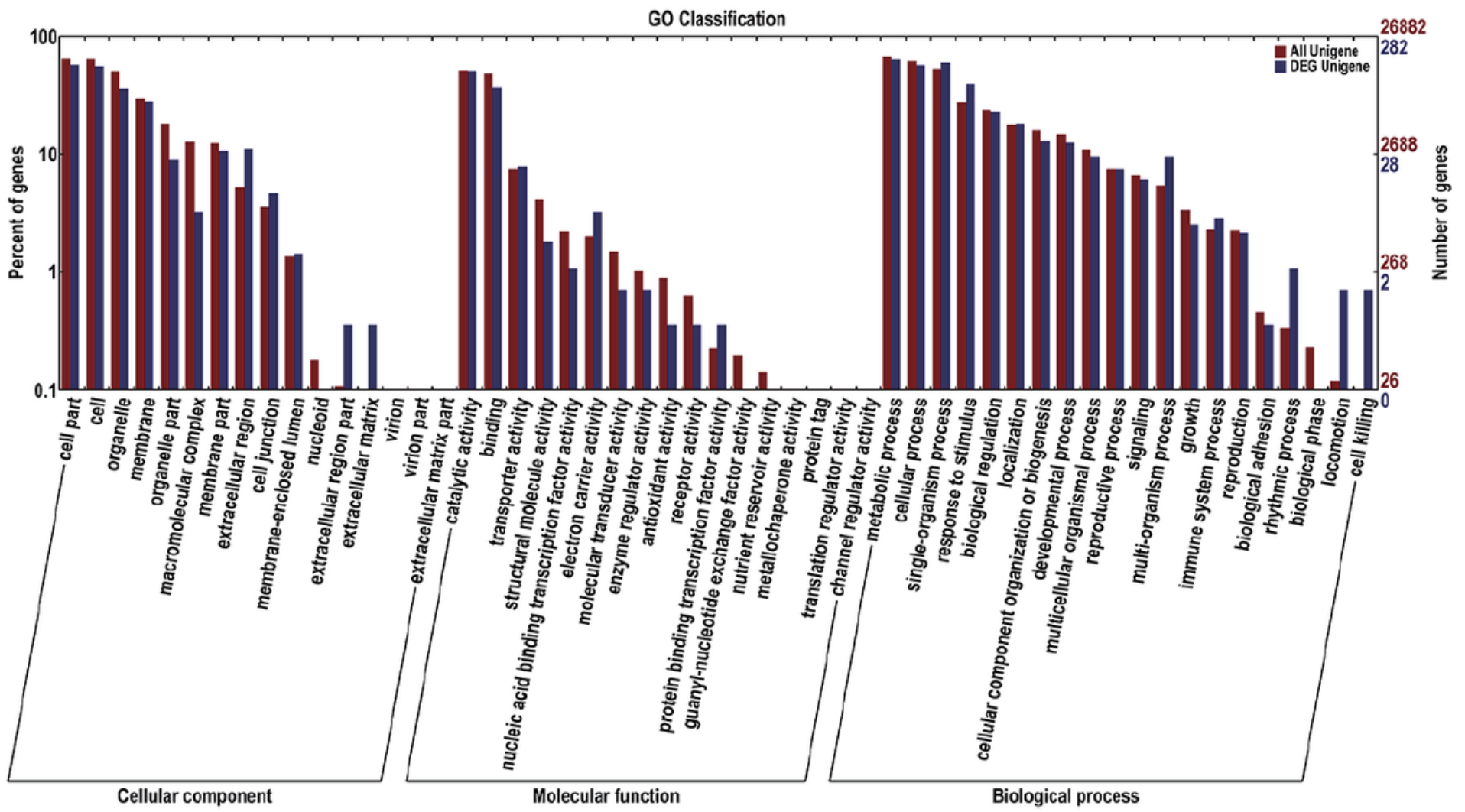


\section{Figure 5}

Clustering diagram of enriched GO terms from 651 annotated differentially expressed genes (DEGs). Genes were assigned into three main categories: biological processes, cellular components or molecular functions.

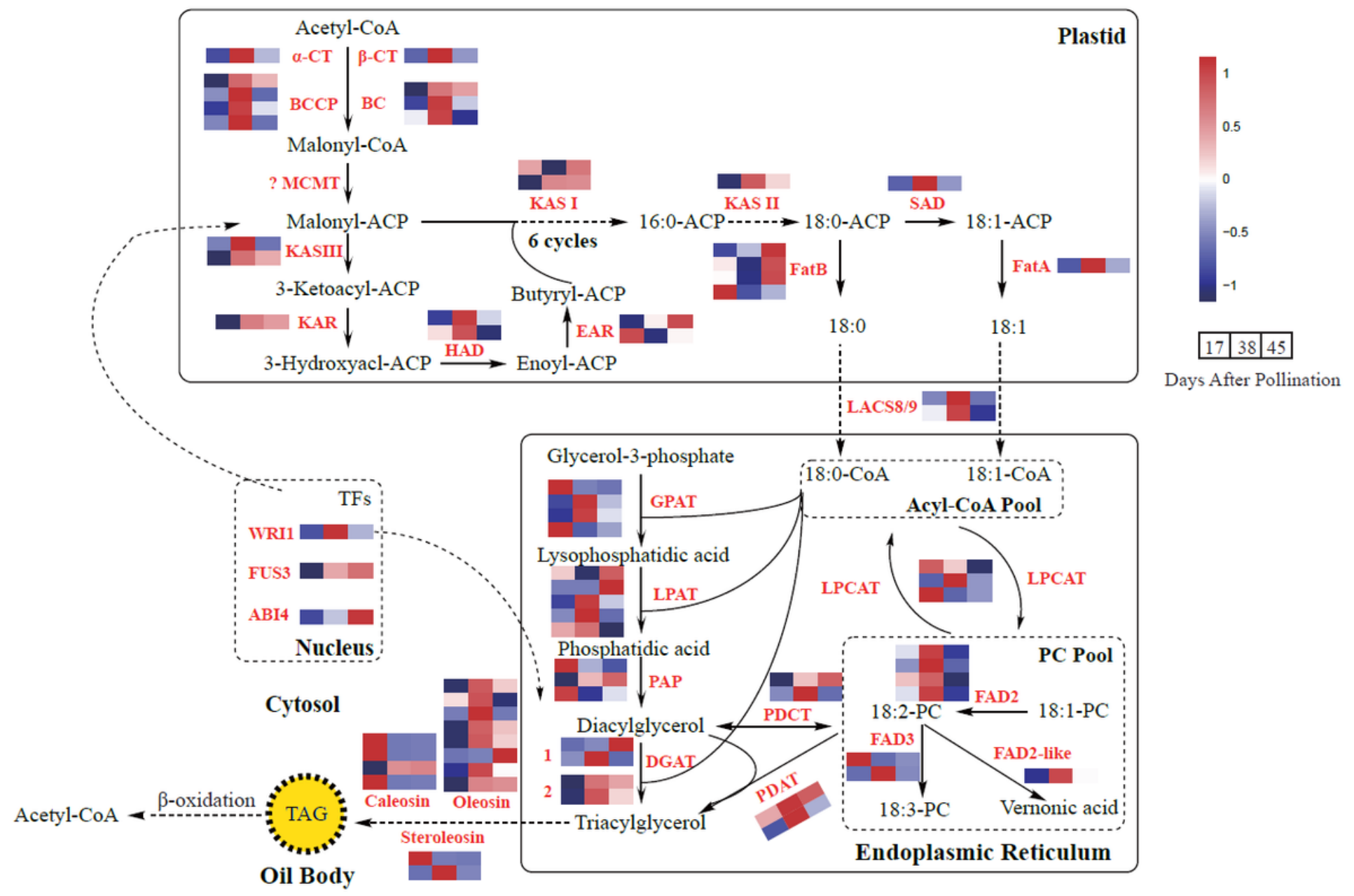

\section{Figure 6}

The proposed fatty acid and TAG biosynthetic pathways in V. galamensis seeds. The reconstructed pathway included the fatty acids de novo biosynthesis, fatty acid modification, triacylglycerol (TAG) assembly and storage process. The heatmap showed the RPKM value of each unigene in the initial, fast and final oil accumulation stages. Abbreviations are listed in Additional file 4. 
a

RcFAH12

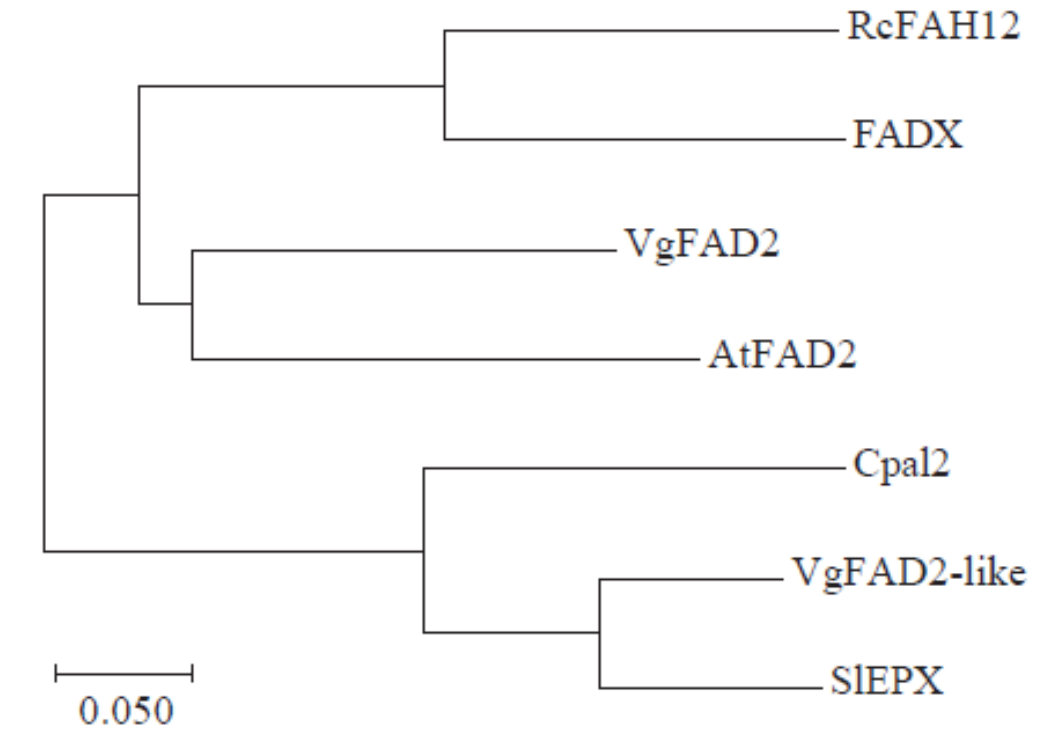

$\mathrm{b}$

$>$ VgFAD2-like

MMMSDSCDDHDQLVKDDHDINERAPVDAAPFSLSDLKKAIPPHCFQRSAIRSSCYVVQDL

IITFLLYTLANSYIPLLPPPLPYFAWPVYWFCQSSILTGLWVIGHECGHHAYSEYQWVDNTV HIS-BOX1

GFILHSLLLTPYFSWKYS HRKHHANTNSLENEEVYIPKAKSQLRNYSNFKFLDNTPGRIFIL HIS-BOX2

LIMLTLGFPLYLLTNISGKKYQRFANHFDPLSPIFSERERIQVVLSDVGLIAVFYGLKFLVAK

KGFGWVMRMYGAPVVGLNAFIIMITYLHHTHLSSPHYDSTEWNWIKGALTTIDRDFGLL

NRVFHDVTHTHVLHHLFPYIPHYHAKEASDAIKPVLGEYRMIDRTPFYKAMWREAKECI HIS-BOX 3

YIEPDEDKKHKGVYWYHKM

\section{Figure 7}

Characterization of FAD2-like protein in V. galamensis. (a) The position of three conserved histidine boxes in FAD2-like protein sequence. (b) Phylogenetic tree of VgFAD2-like and other divergent form of FAD2 sequences. The tree was generated by ClustalW, Neighbor-Joining ( $N J$ ) method was adopted. Sequences used to construct the tree included Arabidopsis thaliana oleate desaturase AtFAD2 (NM_112047), Vernonia galamensis oleate desaturase VgFAD2 (AF188264), Ricinus communis hydroxygenase RcFAH12 (U22378), Vernicia fordii conjugase FADX (AF525535), Crepis palaestina epoxygenase Cpal2 (Y16283), and Stokesia laevis epoxygenase SIEPX (AY462108). 

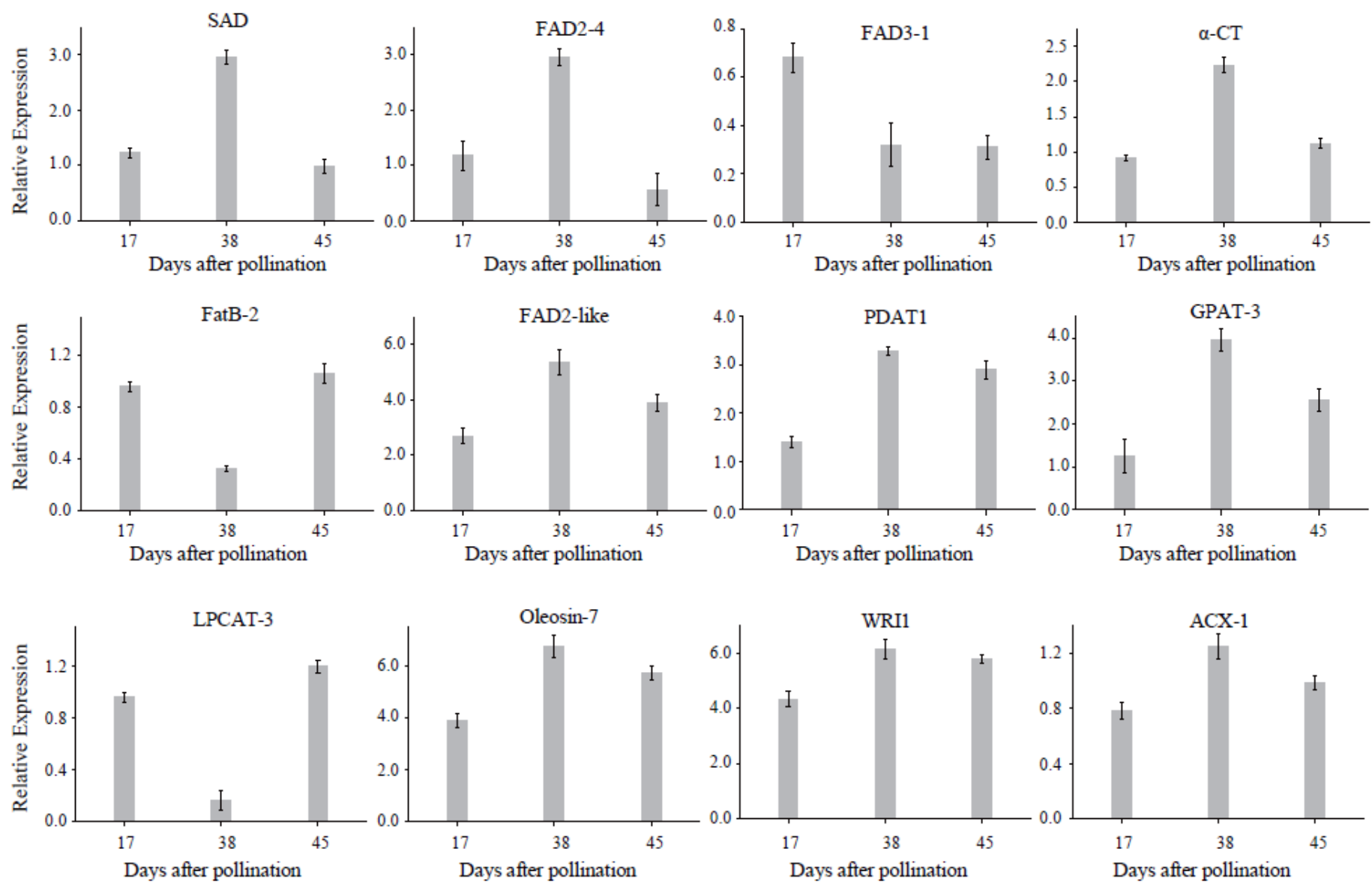

Figure 8

Relative expression analysis of fatty acid synthesis genes in V. galamensis seeds at different developmental stages.

\section{Supplementary Files}

This is a list of supplementary files associated with this preprint. Click to download.

- Tables1.xls

- Tables2.xls

- Tables3.xls

- Tables4.xls

- Tables5.xls

- Tables6.xls 